\title{
ANALYSING THE AUDIO WATERMARKING SCHEMES AND FEATURES
}

\author{
Electa Alice Jayarani A. ${ }^{1}$, D. Ane Delphin ${ }^{2}$, Ambily Babu ${ }^{3}$ \\ ${ }^{1}$ Assistant Professor, Department of ECE, T. John Institute of Technology, Karnataka, India \\ ${ }^{2}$ Assistant Professor, Department of ECE, T. John Institute of Technology, Karnataka, India \\ ${ }^{3}$ Assistant Professor, Department of ECE, T. John Institute of Technology, Karnataka, India
}

\begin{abstract}
In recent years, there is a rapid growth in information technology and multimedia. At the same time information security and copyright has become an issue as piracy and malice manipulation is common. Watermarking is commonly used technique to solve the above mentioned issue. By inserting a watermark in the host audio signal the copyright problem can be solved. As a result the biggest debate in the current era is digital audio watermarking. More affordable the watermarking should be to make the extraction easier. This paper mainly discusses on audio watermarking principle, features of watermarking, performance evaluation techniques and research gap on audio watermarking.
\end{abstract}

Keywords: Audio Watermarking, Features of Watermarking, Performance Evaluation Techniques

\section{INTRODUCTION}

Digital watermarking is embedding a permanent watermark into digital media and retrieve the watermark to prove the authenticity [1][ 21]. Digital media can be Image, audio, video and text documents. The digital audio files are mostly commonly shared in open environment as it can be compressed and transmitted. Hence the research on digital audio watermarking (DAW) is highlighted recently [1].

The crucial factors of DAW compare to image and video watermarking are:

1. The Human Auditory System (HAS) can detect a slight changes in amplitude and frequency of signal precisely. Thus HAS is very delicate than the Human Visual System (HVS) [1][2][26]. The HAS is very much perceptive to the additive white Gaussian noise (AWGN), which can be detected if the disposition level is low as 70dB. [1][2][26].

2. The watermark capacity is much lower than image and video media, because the acoustic signals are represented by smaller amount of bits per time interval [1][2][26].

\subsection{Audio Watermarking Requirement}

The requirements of DAW based on the International federation of the Phonographic Industry (IFPI) are as follows [3]:

1. The perception quality of the host signal should not be damaged.

2. Signal to Noise ratio(SNR) $>20 \mathrm{~dB}$

3. The watermark capacity should be more than $20 \mathrm{bps}$

4. DAW should withstand the most typical signal refinement and attacks.
5. DAW should be able to prohibit the unlicensed discovery, removal and embedding, without disturbing the nature of audio [3].

\subsection{Principles of Psychoacoustics:}

Fact finding the statistical likeness between acoustic stimuli and hearing sensations is called sound perception. The Psychoacoustic is defined as the discipline of sound perception. Psychoacoustic model is a quantitative model which could nearly complement the hearing mechanism [1]. $20 \mathrm{~Hz}$ to $20 \mathrm{KHz}$ is the human hearing frequency. $100 \mathrm{~Hz}$ and $8 \mathrm{KHz}$ is the most receptive part of HAS. The hearing range of human is shown in fig 1 . The figure shows the different aural thresholds. The thresholds are represented in terms of sound pressure level (SPL) curves as function of frequency[19,20].

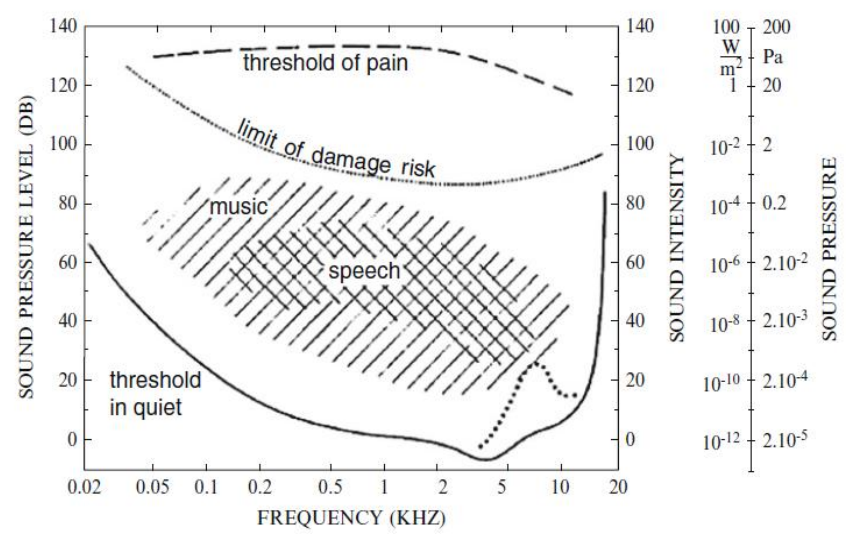

Fig.1. Hearing range

\section{DIGITAL AUDIOWATERMARKING SYSTEM}

The watermarking system consists of two stages. The embedding process is defined as 


$$
\mathrm{S}_{\mathrm{w}}=\text { Embedding }\left(\mathrm{S}_{\mathrm{o}}, \mathrm{W}_{\mathrm{o}}, \mathrm{K}_{\mathrm{w}}, \mathrm{K}_{\mathrm{s}}\right)
$$

Where $\mathrm{S}_{\mathrm{o}}$ is host signal

$\mathrm{W}_{\mathrm{o}}$ is original watermark

$\mathrm{K}_{\mathrm{w}}$ is watermark key

$\mathrm{K}_{\mathrm{s}}$ is secret key

The second stage is extraction process, which is defined as

$$
\mathrm{W}_{\mathrm{e}}=\operatorname{detection}\left(\mathrm{S}_{\mathrm{a}}, \mathrm{S}_{\mathrm{o}}, \mathrm{K}_{\mathrm{w}}, \mathrm{K}_{\mathrm{s}}\right)
$$

Where $\mathrm{S}_{\mathrm{a}}$ is attacked signal

$\mathrm{S}_{\mathrm{o}}$ is host signal (optional)

$\mathrm{K}_{\mathrm{w}}$ is watermark key

$\mathrm{K}_{\mathrm{s}}$ is secret key

\section{FEATURES OF AUDIO WATERMARKING}

\subsection{Imperceptibility}

Imperceptibility is related to the quality of original audio in the presence of watermark. The watermark-embedding algorithm has to insert additional data without affecting the perceptual quality of the audio host signal. The watermarked must be inaudible within the host audio to maintain audio quality [1].

\subsection{Robustness}

Robustness is a scale of invulnerability against signal manipulation and compression. It is measured in terms of correlation factor, which is the similarity between the original watermark and extracted watermark image. The value lies from 0 to 1 . Ideally it should be 1 , but the value above 0.7 is acceptable. The various signal manipulations are Noise addition, Resampling, LPF, Echo addition, Data compression, Random samples cropping Zero inserting, Pitch invariant time stretching, Tempo pressured pitch shifting.

\subsection{Security}

As the watermarked signal is open to social media, the security of audio should be given higher priority. There is no restriction to access the watermarked audio as it available in open environment. The secret key is used as an extra effort to enhance the security.

\subsection{Data Capacity}

The bit rate of the embedded watermark is the number of the embedded bits within a unit of time and is usually given in bits per second (bps). The capacity of the watermark method is tested by increasing the length of the watermarking message. Maximum number of bits to be embedded in host is given as,

$\mathrm{C}=\mathrm{Fs} / \mathrm{N}(\mathrm{bps})$

Where Fs is Sampling frequency

$\mathrm{N}$ is Number of samples per frame.
Depend on application the watermark capacity varies. For example for Ownership application capacity up to $0.5 \mathrm{bps}$, Broadcast application capacity is up to $15 \mathrm{bps}$ and Envisioned application the capacity is up to $150 \mathrm{Kbps}$.

\subsection{Computational Complexity}

Embedding \& extraction speed and the number of embedders and detectors are the two main features for measuring the computational complexity.

The nominal computation pace in embedder is given by

$$
\mathrm{CC}_{\text {embedding }}=\mathrm{N}_{\mathrm{w}} / \mathrm{t}_{\text {embedding }} \text { (bps) }
$$

The nominal computation pace in detector is given by

$$
\mathrm{CC}_{\text {detection }}=\mathrm{N}_{\mathrm{w}} / \mathrm{t}_{\text {detection }}(\mathrm{bps})
$$

Where $\mathrm{t}$ embedding \& $\mathrm{t}_{\text {detection }}$ is embedding and detection time respectively.

\section{PERFORMANCE EVALUATION}

\subsection{Transparency Test}

Transparency Test is a fulfillment quality of perceptual nature. Transparency test can be done by two ways.

\subsubsection{Subjective Test}

The Subjective Listening Test involves the human acoustic perception. ABX test and MUSHRA (Multi Stimuli with Hidden Reference and Anchors) is the Subjective Listening Tests. In this test Different industry people, like, Recording Engineer, Mastering Engineer, Synthesizer Engineer, Audio critics are invited and asked to grade the watermarked audio. The grading is called SDG (Subjective Difference Grade). The SDG lies between 1 and 5. The disadvantages of subjective test are, as human is judging there can be misjudgment, more time and cost is needed to experiment this type of listening test. [1].

\subsubsection{Objective Evaluation Test}

The Objective Evaluation Test is perception modeling or objective quality measures. The various tool are Evaluation of Audio Quality (EAQUAL), Perceptual Evaluation Audio Quality (PEAQ), Perceptual model Quality Assessment (PEMQ-Q). The grading is called ODG (Objective Difference grade). The IFPI standard is shown in table1. 


\begin{tabular}{|c|c|}
\hline Difference Grade & Description of impairment \\
\hline 0 & Imperceptible \\
\hline-1 & Slightly annoying \\
\hline-2 & Annoying \\
\hline-3 & Very annoying \\
\hline-4 & Table1. ODG level \\
\hline
\end{tabular}

\section{AUDIO WATER MARKING TECHNIQUES}

The watermarking can be implemented in time domain and frequency domain. Time domain approach is rather easier to implement and requires less computation. But it is less robust to digital signal attacks. [4-5]In addition to the traditional Fourier transform, various watermarking uses various domain, such as Spread Spectrum, Cepstral transform, Discrete cosine transform(DCT), the wavelet transform (DWT,LWT,..), the modified Complex lapped Transform(MCLP) and Modified discrete Cosine transform.[4-5]. The above mentioned methods mainly focus on Digital channel [23]. In this section, different audio watermarking techniques are surveyed.

\subsection{Phase Coding}

The HAS can perceive only the relative phase not the absolute phase. Therefore the watermark can be embedded into the phase of the original audio file. [1][2][28]

\subsubsection{Algorithm}

Step1: Split the host signal into Np frames with N samples. Step2: Generate the watermark. Here, binary watermark is used

Step3: Calculate the magnitude \& Phase spectrum using fast Fourier transform.

Step4: Phase spectrum is modified according to the watermark.

If watermark bit $=1$ then phase is $\prod / 2$

If watermark bit $=0$ then phase is $\left(-\prod / 2\right)$

Step5: reconstruct the watermarked frame.

Step6: Extract the watermark based on the phase spectrum.

If extracted phase is $\geq 0$ then watermark bit $=1$

If extracted phase is $<0$ then watermark bit $=0$

\subsubsection{Results}

Table 2. Phase coding results

\begin{tabular}{|c|c|c|}
\hline Test & \multicolumn{2}{|c|}{ Measurement } \\
\hline $\begin{array}{c}\text { Perceptual } \\
\text { Quality }\end{array}$ & \multicolumn{2}{|c|}{ SNR/dB is -2.55 } \\
\hline Robustness & Attack & BER \\
\cline { 2 - 3 } & No attack & 0 \\
\cline { 2 - 3 } & Noise & 19.57 \\
\cline { 2 - 3 } & Compression & 18.98 \\
\hline
\end{tabular}

\subsection{Sonic Watermarking}

The challenge in watermarking field is embedding the watermark in real time not in digitally stored file. The sonic watermarking is proposed algorithm for live performance [27]. In sonic watermarking, the watermark sound generated by a watermark generator is mixed with the host sound in the air.

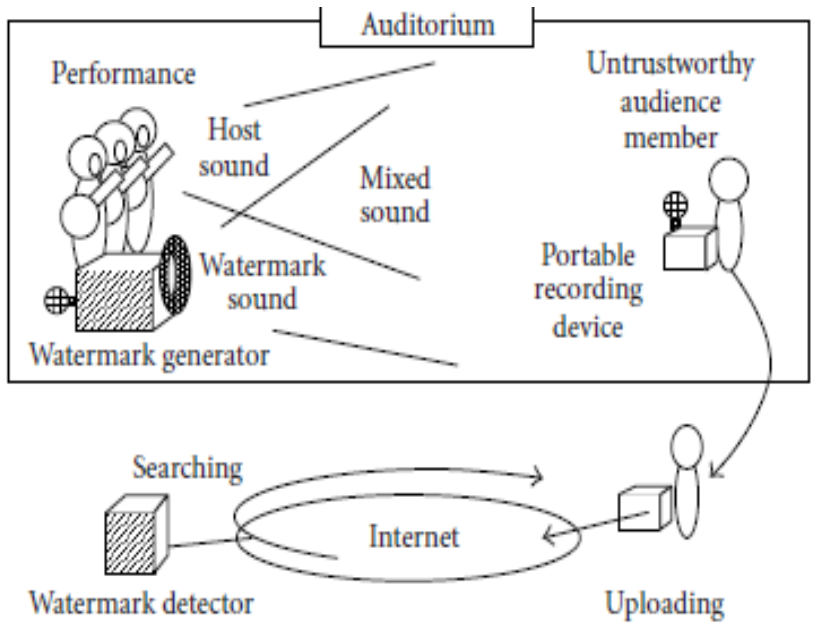

Fig.2. Sonic watermarking system

According to the author the problems in sonic watermarking are as follows:

- Real time embedding: As the watermark and host is mixed in air, there can be delay.

- Robustness: Reverberation, noises made by audience are the next major drawbacks for live embedding.

- Acoustic quality: The Strength and different location of sound sources also affect the embedding strength.

Main concern in live watermarking is Analog and digital inter conversion taking place before and after embedding the watermark. The MATLAB simulation diagram of audio signal before and after conversion is shown in fig3.
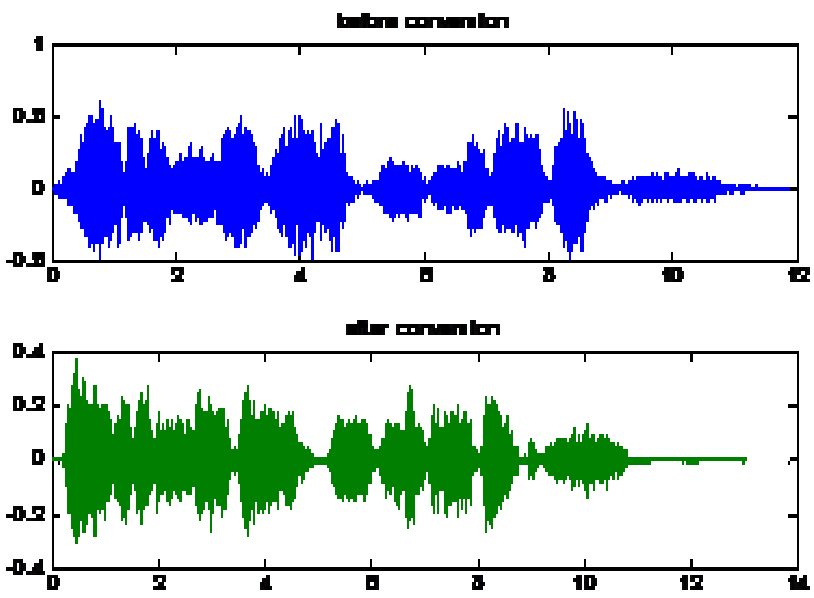

Fig.3. Watermarked signal before and after conversion

In real time application of audio watermarking, the watermarked signal is sent to an analog output and digitized again. Thus the watermarking algorithm should be robust 
against DA/AD conversion. There are three types of such conversion of cable - based audio transmission, radio broadcasting and air- based broadcasting. There are few algorithms for cable-based audio transmission.[6-7] But the air-based broadcasting or contactless watermarking in an acoustic space has not been developed.

\subsection{Analog and Digital Inter Conversion}

The Analog and Digital Inter conversion module should consider the following:

- The audio frequency $>50 \mathrm{~Hz}$ should be taken into account of watermarking.

- The noise in DA/AD process such as Quantization distortion and filtering process. Embedding should not use the high frequency also because of the above mentioned noise in $\mathrm{DA} / \mathrm{AD}$ process.

- The record time may be earlier or later than the play time, so the starting position of the watermark should have synchronized signal.

- Analog and Digital Inter conversion for the different sound cards.

\section{CONCLUSIONS}

Various audio watermarking algorithms are proposed to prevent the violations in use of audio files, the enforcement of ownership management, etc. In this paper, we have analyzed the phase audio watermarking. This watermarking can't be used for live embedding. The next algorithm discussed is sonic watermarking. The non cable based audio watermarking has not been developed and open to research.

\section{REFERENCES}

[1]. Y.Lin and W.H.Abdulla, "Audio Watermark: A Comprehensive Foundation Using MATLAB", Springer International Publishing Switzerland 2015.

[2]. W. Bender, D. Gruhl, and N. Morimoto, "Techniques for data hiding", Proc. SPIE, vol. 2420, pp.40 1995 .

[3] Prayoth Kumsawat "A genetic algorithm optimizationtechnique for multiwavelet-based digital audio watermarking", EURASIP Journal on Advances in Signal Process., pp.471842 -471852, 2010.

[4]Hwai-Tsu $\mathrm{Hu}$, Hsien-Hsin Chou, "Incorporation of perceptually adaptive QIM with singular value decomposition for blind audio watermarking", EURASIP Journal on Advances in Signal Processing 2014 ,2014:12.

[5] Arnold, M.; Xiao-Ming Chen; Baum, P.; Gries, U.; Doerr, G,"A Phase Based Audio Watermarking System Robust to Acoustic Path Propagation", IEEE Transactions on Information Forensics and Security, vol.9, no.3, pp.411425, March 2014.

[6] Xiaoming Zhang; Zhaoyang Yu, "Cepstrum-Based Audio Watermarking Algorithm against the A/D and D/A Attacks," Fifth International Conference on Information Assurance and Security, 2009. IAS '09. , vol.1, pp.740-743, 18-20 Aug. 2009.

[7] Cairong Li; Ruimin $\mathrm{Hu}$; Wei Zeng, "Radon transform and DWT based audio watermarking algorithm against DA/AD conversion," International Conference on Audio,
Language and Image Processing (ICALIP), 2012, pp.282286, 16-18 July 2012.

[8] Qijun Guo; Yanbin Zhao; Pingpan Cheng; Fengming Wang, "An audio digital watermarking algorithm against $\mathrm{A} / \mathrm{D}$ and D/A conversions based on DCT domain," 2nd International Conference on Consumer Electronics, Communications and Networks (CECNet), 2012, pp.871876, 21-23 April 2012.

[9] Steinebach, M.; Lang, A.; Dittmann, J.; Neubauer, C., "Audio watermarking quality evaluation: robustness to DA/AD processes," International Conference on Information Technology: Coding and Computing, 2002. Proceedings., pp.100-103, 8-10 April 2002.

[10] Hering, H.; Hagmuller M; Kubin, G., "Safety and security increase for air traffic management through unnoticeable watermark aircraft identification tag transmitted with the VHF voice communication," The 22nd Digital Avionics Systems Conference, 2003. DASC '03., vol.1, pp.4.E.2-41-10 vol.1, 12-16 Oct. 2003.

[11] Modegi, T., "Nearly Lossless Audio Watermark Embedding Techniques to be Extracted Contactlessly by Cell Phone," 7th International Conference on Mobile Data Management, 2006. MDM 2006., pp.36-36, 10-12 May 2006.

[12] Irena Orovic and Srdjan stankovic, “ Time - Frequency - Based Speech Regions Characterization and Eigen value decomposition Applied to Speech Watermarking", EURASIP Journal on Advances in Signal Processing, Volume 2010,Article ID 572748,10 pages.

[13] Khaldi, K.; Boudraa, A., "Audio Watermarking Via EMD,"IEEE Transactions on Audio, Speech, and Language Processing, , vol.21, no.3, pp.675-680, March 2013.

[14] Fallahpour, M.; Megias, D., "Audio Watermarking Based on Fibonacci Numbers," IEEE/ACM Transactions on Audio, Speech, and Language Processing, , vol.23, no.8, pp.1273-1282, Aug. 2015.

[15] Takahashi, A.; Nishimura, R.; Suzuki, Y., "Multiple watermarks for stereo audio signals using phase-modulation techniques," IEEE Transactions on Signal Processing, , vol.53, no.2, pp.806-815, Feb. 2005.

[16] Guang Hua; Goh, J.; Thing, V.L.L., "Time-Spread Echo-Based Audio Watermarking With Optimized Imperceptibility and Robustness," IEEE/ACM Transactions on Audio, Speech, and Language Processing, , vol.23, no.2, pp.227-239, Feb. 2015.

[17] Malik, H.M.A.; Ansari, R.; Khokhar, A.A., "Robust Data Hiding in Audio Using Allpass Filters," IEEE Transactions on Audio, Speech, and Language Processing, , vol.15, no.4, pp.1296-1304, May 2007.

[18] Shijun Xiang, "Audio watermarking robust against D/A and A/D conversions", EURASIP Journal on advances in signal processing, volume 2011, Dec 2011.

[19] E.Zwicker,H.Fastl, "Psychoacoustics: Facts and models", Springer Science \& Business Media, 30-Jun-2007 - science- 463 pages.

[20] M.Bosi,R.E.Goldberg, "Introduction to digital audio coding and standards", The Springer International series in Engineering and Computer Science, Kluwer Academics publishers, 434 pages ,2003. 
[21] Pooya Monshizadeh Naini, "Digital Watermarking Using MATLAB, Engineering Education and Research Using MATLAB”, Dr. Ali Assi (Ed.), ISBN: 978-953-307656-0, InTech open access publisher, 2011.

[22] J. Blackledge, F. Omar, "Audio Data Verification and Authentication Using Frequency Modulation Based Watermarking", International Society for Advanced Science and Technology, Journal of Electronics and Signal Processing, Vol. 3, Issue 2, pp. 51-63, 2008.

[23] Cvejic, Nedeljko, and Tapio Seppänen. "Digital Audio Watermarking Techniques and Technologies: Applications and Benchmarks." IGI Global, 2008. 1-344.

[24] Xing He, "Watermarking in Audio: Key Techniques and Technologies", Cambria Press, January 28, 2008,182 pages.

[25] Pranab Kumar Dhar,Tetsuya Shimamura, "Advances in Audio Watermarking Based on Singular Value Decomposition", Springer International Publishing,2015,58pages.

[26] Hartung, F.; Kutter, M., "Multimedia watermarking techniques," in Proceedings of the IEEE, vol.87, no.7, pp.1079-1107, Jul 1999.

[27] Ryuki Tachibana; "Audio watermarking for live performance", Proc. SPIE 5020, Security and Watermarking of Multimedia Contents V, 32, June 13, 2003.

[28] M.Arnol, M.Schmucker, S.D.Wolthusen, Techniques and Application of Digital Watermarking and content protection (Artech House,Boston,2003)

\section{BIOGRAPHIES}

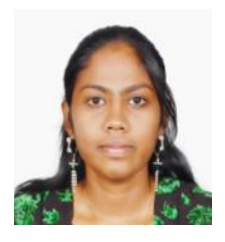

Electa Alice Jayarani. A is currently working as Assistant Professor, Electronics and Communication Department in T.John Institute of Technology, Karnataka, India. She is doing her research in audio watermarking.

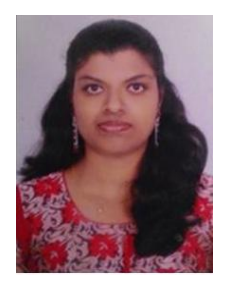

D. Ane Delphin is an Assistant Professor in the Department of ECE at T. John Institute of Technology. She has 5 years of teaching experience. She is currently pursuing her $\mathrm{Ph}$. D. in VIT University, Vellore. Her areas of interest are VLSI design and Image Processing.

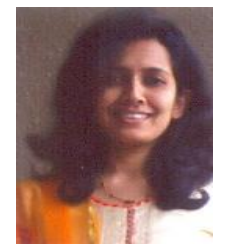

Ambily Babu is currently working as Assistant Professor, Electronics and Communication Department in T.John Institute of Technology, Karnataka, India. She has 3 years of teaching experience. 